\title{
The zygoma anatomy-guided approach (ZAGA) for rehabilitation of the atrophic maxilla
}

\author{
Carlos Aparicio ${ }^{1}$ D . Antonio Olivo ${ }^{2}$ - Victor de Paz $^{3}$. Daniel Kraus ${ }^{4}$. \\ Manuel Martin Luque ${ }^{5} \cdot$ Eduardo Crooke $^{6} \cdot$ Peter Simon $^{7} \cdot$ Madalina Simon $^{7}$. \\ Jose Ferreira $^{8}$. Andre Sakima Serrano ${ }^{9}$ Jan Peter $~_{1 g^{9}}$. Arturo Bilbao ${ }^{10}$. \\ Alberto Fernandez ${ }^{11}$. Pedro Guitián ${ }^{12}$. Jay Neugarten ${ }^{13}$
}

Received: 19 June 2021 / Accepted: 31 December 2021 / Published online: 23 February 2022

(c) The Author(s) 2022

\begin{abstract}
A protocol to perform a prosthetically driven minimally invasive zygomatic osteotomy, named zygoma anatomy-guided approach (ZAGA) is introduced. The ZAGA method aims at promoting a patient-specific therapy by adapting the osteotomy type to the patient's anatomy. In most cases, this method avoids the opening of a window or slot into the lateral wall of the maxillary sinus before implant placement. Instead, a mucoperiosteal flap, including the posterior maxillary wall and the superior zygomatic rim, is raised to allow visual control of the complete surgical field. The surgical management of the implant site is guided by the anatomy of the patient according to specific prosthetic, bio-mechanic, and anatomic criteria. The ZAGA Concept represents the logical evolution of the extra-sinus technique and ZAGA classification previously described by Aparicio. The results of using the combination of the ZAGA Concept together with the new ZAGA implant designs consistently show less traumatic osteotomy; better implant stability; improved bone to implant contact, and bone sealing around the implant neck. Additionally, the rate of late complications such as oral-sinus communication or soft tissue recession dramatically decreases when compared to the original technique.
\end{abstract}

Keywords ZAGA · ZAGA Flat · ZAGA Round · Tunnel osteotomy · Channel osteotomy

Carlos Aparicio

carlos.aparicio@zagacenters.com

Extended author information available on the last page of the article 


\section{Quick reference/ Description}

In patients with a resected or atrophic maxilla, placement of traditional endosseous implants is difficult or even not possible due to inadequate bone architecture requiring the use of zygomatic implants. In most scenarios, patients with extreme maxillary atrophy have only one chance for oral fixed rehabilitation using zygomatic implants as prosthetic anchorage. The zygoma anatomy-guided approach concept individualizes the surgical procedure according to the patient's anatomy and aids the surgeon to determine an ideal prosthetic implant position along with proper sinus sealing while also overcoming the drawbacks of the previous surgical approaches.

\section{Indications}

Rehabilitation of severe maxillary atrophy.

\section{Materials/ Instruments}

- Modified dissectors (Salvin Instruments ZAGA Kit)).

- Modified retractor with a distal hook (Salvin Instruments ZAGA Kit)).

- Zygomatic burs (Versah ZGO Kit).

- Zygomatic round bur (Straumann).

- Zygomatic twist drill (Straumann).

- Modified zygomatic implant: ZAGA Round (Straumann ZAGA Round).

- Modified zygomatic implant: ZAGA Flat (Straumann ZAGA Flat).

- 3-D models for surgical rehearsal: https://zagacenters.com/get-a-3d-model/

\section{Procedure}

\section{The zygomatic procedure and its evolution}

In patients with a resected or atrophic maxilla, placement of traditional endosseous implants is not possible due to inadequate bone. The management of a severely atrophic maxilla using zygomatic implants is different and more complicated than the placement of conventional endosseous implants from a surgical perspective. In most cases, patients with extreme maxillary atrophy have only one chance for oral prosthetic rehabilitation using zygomatic implants. The learning curve of the procedure may be long due to the reduced number of patients to be treated in a single office. Hence, the management of maxillary atrophy using zygomatic implants should preferably be performed by an experienced clinician. 
P-I Brånemark introduced the original surgical technique (OST) characterized by a palatal entrance and an intra-sinus path of the implant body until its zygomatic anchorage and two-stage surgery. However, it had drawbacks such as frequent bulky prostheses and oro-antral communication, which needed to be overcome.

\section{First variations of the original technique}

Stella and Warner in 2000 followed by Peñarrocha et al. explained the sinus slot technique that utilizes a slot window through the buttress wall of the maxilla for visibility of the implant insertion. It also emphasized the importance of placing the zygoma platform directly over the alveolar ridge. The Stella-Warner approach demonstrated a marked improvement of the original zygomatic implant insertion technique, but it still did not intend to solve complications as the oral-antral communication.

In 2003, Boyes-Varley and colleagues modified the OST with the aim of establishing improved access to the surgical site and reducing post-operative morbidity. They also modified implant head angulation into a $55^{\circ}$ correction.

Aparicio and cols. described 1-year experience with a novel extra-sinus surgical technique for zygomatic implant insertion at the Europerio 2005 meeting whose procedures were published in 2006. Miglioranza and colleagues also introduced an approach for placement of zygomatic implants in an exteriorized manner in 2006 in Portuguese. Problems related to the extra-sinus technique as buccal soft tissue recession appeared when the technique was used as the rule for all types of patients.

In 2011 and 2012, Aparicio and cols described the zygoma anatomy-guided approach (ZAGA) as a novel protocol developed to overcome the drawbacks of the original technique as well as the extra-sinus approaches.

\section{The zygoma anatomy-guided approach (ZAGA)}

ZAGA, the acronym for zygoma anatomy-guided approach, is the concept used to place zygomatic implants in a prosthetically driven manner and according to the anatomy of the patient. The ZAGA method aims at promoting a patient-specific therapy by adapting the osteotomy type and implant design to the patient's structure. The technique relies on the recognition of the existence of the inter-individual anatomical differences as well as the intra-individual variations (Fig. 1). In most cases, as explained further, this method avoids the opening of a window or slot into the lateral wall of the maxillary sinus before implant placement. Instead, a mucoperiosteal flap, including the posterior maxillary wall and the superior zygomatic rim, is raised to allow visual control of the complete surgical field (Fig. 2a, b).

The essence of the ZAGA philosophy is to individualize the surgical procedure that is guided by the anatomy of the patient according to specific prosthetic, biomechanic, and anatomic criteria. The ZAGA Concept helps the surgeon to understand the anatomic variations and, accordingly, to determine the ideal prosthetic implant position together with optimized anchorage, proper load distribution, and 
The ZAGA Classification and the Anatomy-Adapted Implant Design

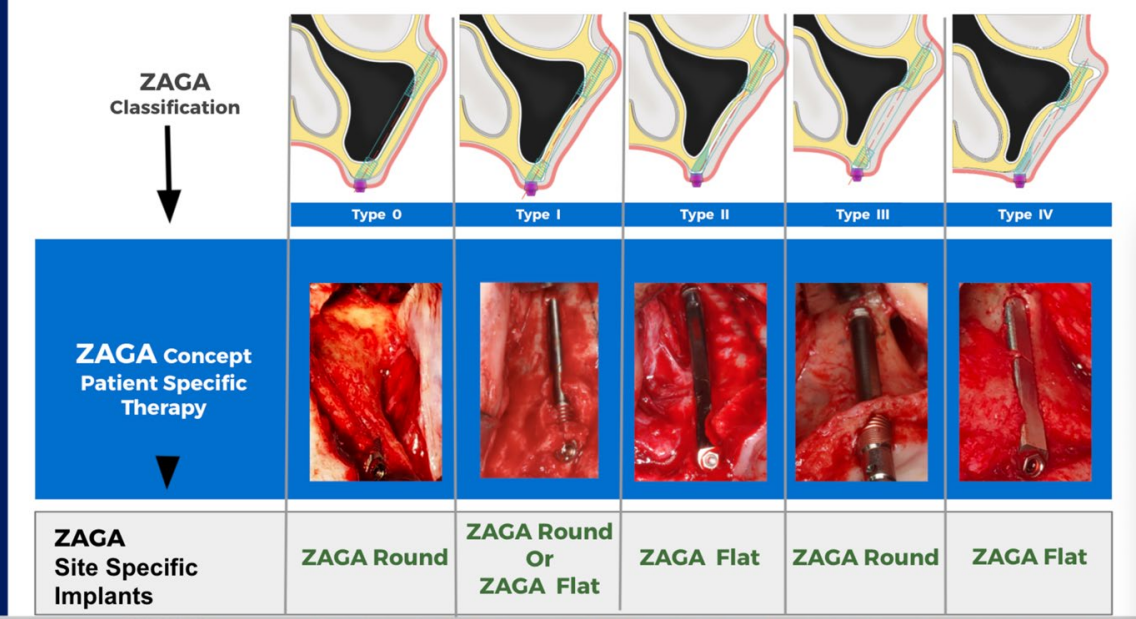

Fig. 1 Upper part, schematic representation of the five possible types of trajectories of the zygomatic implant as per the ZAGA classification for the maxillary wall and alveolar crest. The middle part, clinical images of the individualized implant trajectory as a function of the different anatomies. In the lower part, site-specific implant designs are chosen according to implant trajectory

to achieve crucial long-term objectives of proper bone sinus sealing and stable soft tissue around the implant.

\section{Rationale}

As an evolution of the extra-sinus approach, the relationship of the zygomatic buttress-alveolar crest area was classified into five different types in 2011 (Fig. 1). This classification was used to establish an ideal patient-specific implant path and to develop the zygoma anatomy-guided approach (ZAGA) protocol. The ZAGA Concept provides surgeons with a standardized guiding system to achieve an ideal prosthetic implant position for anchorage, load distribution, and implant head emergence along with the achievement of long-term soft tissue stability and appropriate sinus sealing. The rationale for the ZAGA technique is to give customized treatment to every patient depending on their individual anatomical presentation. The variations of the anatomy make necessary the use of different types of implant designs. Accordingly, two types of new zygomatic implant design and section are introduced: the ZAGA Round used for the ZAGA Tunnel-Type osteotomy and the ZAGA Flat indicated for the ZAGA channel-type osteotomy (Fig. 1).

In the ZAGA technique, the anatomy of the zygomatic bone, maxilla, and maxillary sinus governs the implant site preparation. The anatomical, biomechanical, and prosthetic factors determine the coronal entry point at the level of the 

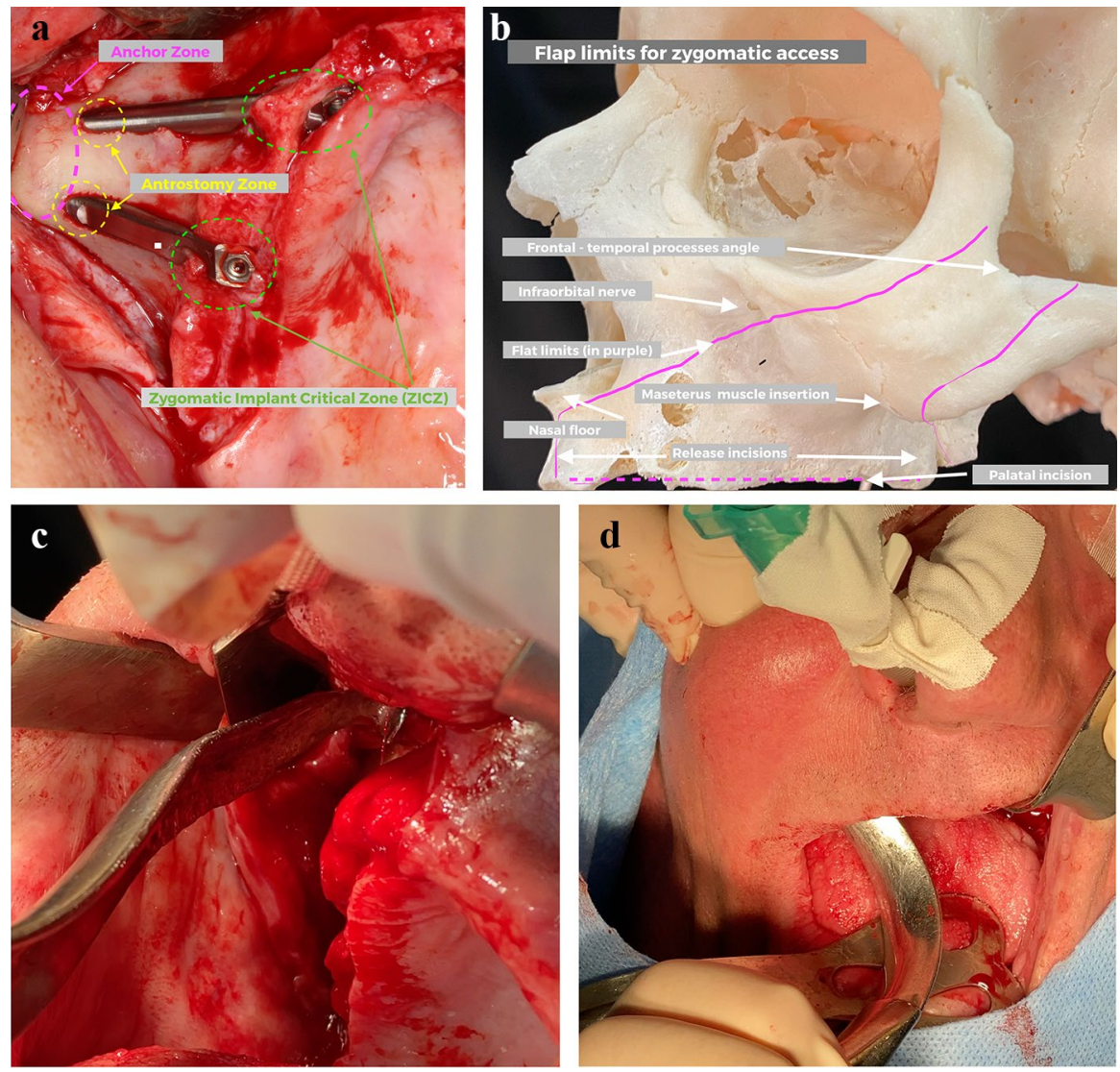

Fig. 2 a The incision runs from the buccal side towards the palatal of the tuberosity, continuing on the palatal side of the ridge in a beveled way up to the middle line. A perpendicular releasing incision to the nasal spine is made. Note the two classic types of osteotomies in 'ZAGA channel' and in 'ZAGA tunnel' for the Straumann ZAGA Flat and the Straumann ZAGA Round designs, respectively. The ZAGA zones are marked with circles as follows: zygomatic implant critical zone (ZICZ) in green; zygomatic antrostomy zone in yellow; zygomatic anchor zone in purple. b The anatomical limits of the recommended flat are marked in purple color. c While maintaining separation from the periosteum, the modified retractor tip will follow the posterior border of the maxillary wall touching the bone until arriving at the superior rim. d The retractor hook was directed between the surgeon's two fingers and placed in the zygomatic angle formed by the frontal and temporal processes

alveolar process to achieve an optimal prosthetic outcome. The zygomatic bone anatomy and the required number and design of implants govern the zygomatic entrance point. These two points define the implant trajectory, which in turn determines the preparation and path of the implant body. In the ZAGA technique, the path of the implant body can range from a total extra-sinus to a total intrasinus path. Indeed, the type of the implant path will determine the election of the implant design (Fig. 1). 


\section{ZAGA surgical access}

The pre-surgical evaluation for the ZAGA protocol is the same as that for the original surgical technique. General anesthesia or intravenous sedation with local anesthesia can be used while treating the patient. One hour before the surgical procedure, antibiotics (amoxicillin $35 \mathrm{mg} / \mathrm{kg}$ ) are administered to the patient in a single dose. In the presence of mandibular teeth, the patient is prescribed additional amoxicillin $750 \mathrm{mg}$ every $8 \mathrm{~h}$ from the day of the surgery for 7 days.

A slightly beveled palatal incision is made starting from the posterior buccal aspect of the maxillary tuberosity till the midline. An ascending vertical vestibularreleasing incision is performed till the nasal spine. The palatal positioning objective is the prevention of eventual soft tissue dehiscence by displacing the adequate volume of connective tissue from the palatal to the buccal (Fig. 2a,b). A mucoperiosteal flap (Fig. 2b) is elevated with modified dissectors (Fig. 2c,d) (Salvin Instruments, ZAGA kit) to expose the alveolar crest, the infraorbital nerve, and the posterior and lateral maxillary walls up to the superior rim of the zygomatic arch. For a less traumatic insertion of unilateral zygomatic implants, a hemi-maxillary flap is advised. When the placement of regular implants is planned below the nasal cavity, the floor of the cavity should be raised to protect the integrity of its mucosa. A modified retractor (Salvin Instruments, ZAGA kit) with a distal hook is anchored onto the superior rim of the zygomatic arch for the following reasons (Fig. 2b-d):

- to ensure good visibility of the zygoma and its boundaries;

- to aid the operator to visualize the appropriate implant direction;

- to guard soft tissues during the most apical second perforation of the anterior zygomatic cortex.

\section{The ZAGA zones}

With a didactic intention, we differentiate three main zones of the zygomatic implant path (Fig. 2a):

- the "zygomatic implant critical zone";

- the "zygomatic antrostomy zone";

- the "zygomatic anchor zone".

The "zygomatic implant critical zone" (ZICZ) is the complex formed by maxillary bone, soft tissue, and the zygoma implant at the coronal level where the first contact with maxillary bone occurs. Residual alveolar bone and soft tissue preservation/augmentation at the coronal level of the zygomatic implant are critical to prevent late complications.

The zygomatic antrostomy zone (ZAZ) is the area where the drill penetrates into the maxillary sinus cavity. Depending on the maxillary anatomy, the zygomatic antrostomy zone will be located either at the internal side of the remaining alveolar 

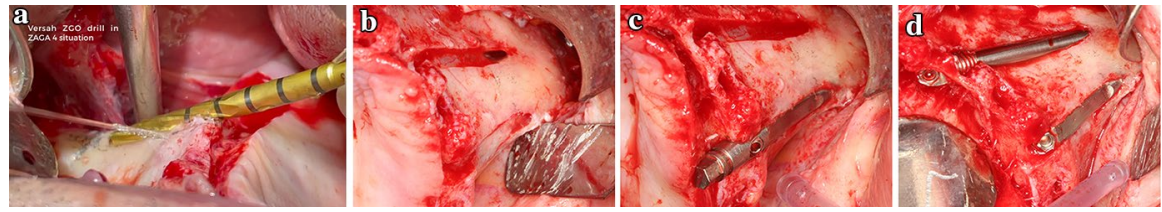

Fig. 3 a The channel-type osteotomy in a ZAGA 4 maxilla is started using the ZGO Kit from Versah. com. b Implant osteotomy aiming to match the implant bed to the implant shape. Note the maximum amount of pristine bone maintained along with membrane integrity without penetrating the antral cavity till it reaches the zygomatic arch. c A Straumann ZAGA Flat zygomatic implant design was inserted. Note the ZAGA Flat sunk down to the crestal level. This positioning prevents soft tissue compression by the implant. d Classic disposition for two zygomatic implants on the same side. Note that the entrance to the maxillary sinus cavity is located at the zygomatic level. The implant itself is responsible for bone sealing

bone (Tunnel Osteotomy) or apically from the ZICZ when there is not enough alveolar bone and the osteotomy trajectory is buccally offset (channel osteotomy). The location of the antrostomy will depend on the zygoma buttress curvature and on the position of the coronal entrance point.

The zygomatic anchoring zone (ZAZ) is the section of the zygomatic bone where the implant reaches its maximal primary stability. To maximize primary stability, the ZAGA Concept uses a tangential zygomatic bone-to-implant intersection, penetrating the four corticals of the maxillary zygomatic process and zygomatic bone to achieve optimum structural zygomatic stabilization.

\section{ZAGA steps and criteria to determine the optimal implant position}

The main aim of performing an osteotomy for conventional or zygomatic implant placement is to establish an implant bed that fits as closely as possible to the implant shape without injuring the adjacent structures. The requirement for a lateral window is eliminated when the osteotomy matches the implant direction (Fig. 3). This preserves maximum bone at the maxillary lateral wall and at the crest. It is the responsibility of the zygomatic implant to close the osteotomy. This osteotomy closure eliminates the need for grafts and makes the surgery minimally invasive. A threestep technique is used to select the ideal implant position immediately following the incision and elevation of the mucoperiosteal flap while adhering to the ZAGA guidelines.

- A. Determine the ideal position of the implant head at the coronal level.

- B. Select the entry point at the zygomatic bone.

- C. Connect the two points.

Each step is performed as per the criteria mentioned below.

\section{A. The oral coronal implant location}


The initial step of implant placement is the identification of the intraoral coronal entrance point. It is governed by anatomic, biomechanical, and prosthetic considerations. As a natural prosthesis is the aim of implant rehabilitation, the starting point (implant head emergence) should be at or close to the top of the alveolar ridge crest (Fig. 4) to achieve a less bulky and esthetic prosthetic design that is easy to clean and does not interfere with the tongue.

To establish mid-crestal implant head emergence, placing the entrance point on the palatal aspect of the alveolar ridge is prevalent. The operator should take into consideration the anatomical criteria while determining the position of the implant head on the buccal-palatal orthoradial axis.

When two zygomatic implants are planned, a mesial-distal entrance at the level of the second premolar or first molar region is advised for balanced load distribution and minimal cantilever length. When four zygomatic implants are planned, the anterior implants should be positioned between the lateral teeth to prevent extensive mesial cantilevers (Fig. 5). The zygomatic implants should not be inserted too close or parallel to each other. Too wide implant designs $(5 \mathrm{~mm}$ diameter) can result in close or parallel implant placement and prevent optimal implant distribution.

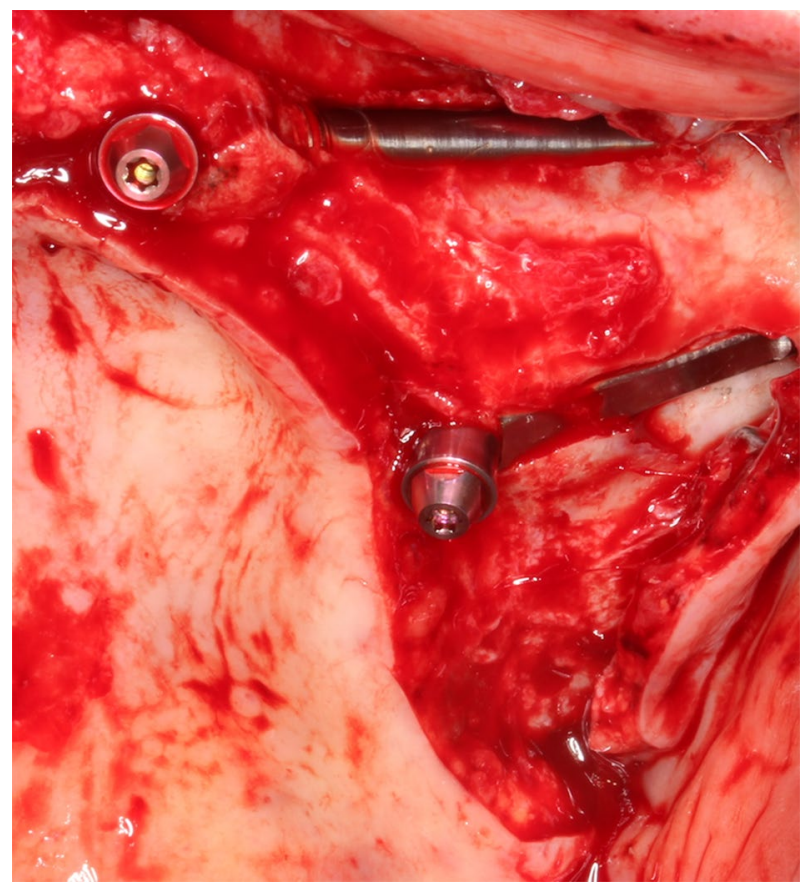

Fig. 4 Implant head emergence close to the ridge crest facilitating an esthetic, non-bulky prosthetic design. The pre-angled $55^{\circ}$ implant head simplifies and expedites the procedure. Note that in most cases a straight abutment can be used. A Straumann ZAGA Round zygomatic implant was placed in the anterior region. Its threaded neck will help to achieve and maintain osseointegration of the alveolar crest. A Straumann ZAGA Flat zygomatic implant was placed in the posterior region. Its flat section would prevent vascular soft tissue compression 


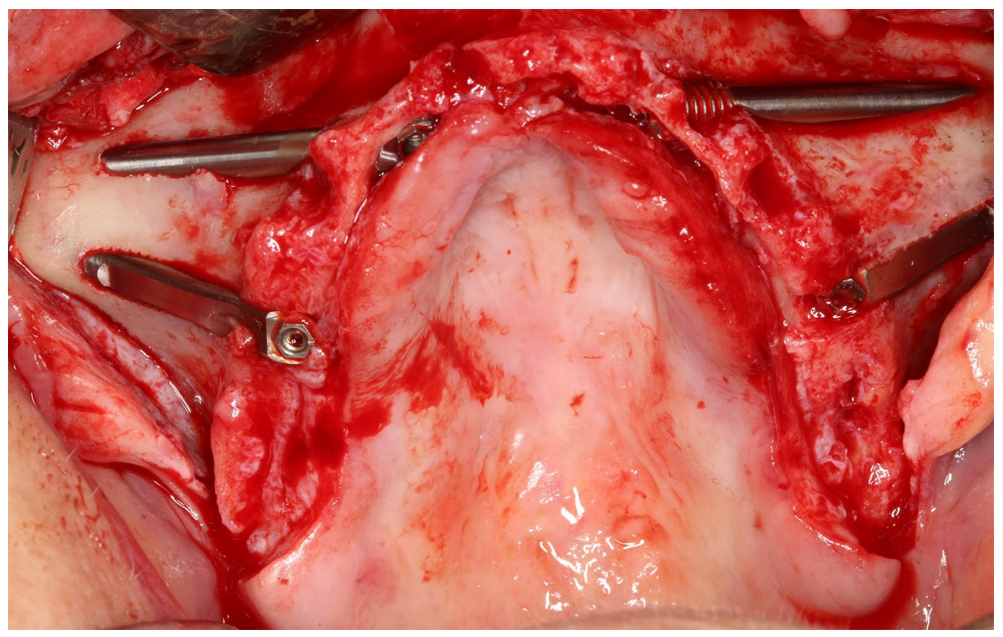

Fig. 5 Typical implant arrangement for 'Quad' procedure as per the ZAGA prosthetically driven concept. Implant heads are located on the alveolar crest of the lateral or canine and second premolar or molar areas

Zygomatic implants should be placed in regions with inadequate bone for the stabilization of conventional implants. The mesial-distal variations of zygomatic implant position should depend on pristine bone distribution. In case of anterior atrophy, the zygomatic implant must be placed in the anterior maxilla (Fig. 6). Two major factors determine the buccal-palatal location of the implant in association with the residual alveolar bone:

- vertical and horizontal resorption of alveolar or basal process;

- anterior maxillary wall curvature.

When there is adequate bone at the sinus floor level to house the implant neck, i.e., $4 \mathrm{~mm}$ high $\times 6-7 \mathrm{~mm}$ wide in a satisfactory alveolar architecture, such as described for original ZAGA types 0 and 1 and some ZAGA 2, the initial osteotomy was done from the palatal aspect of the crest (Fig. 6a). Efforts were made to place the implant through it using a tunnel-shaped osteotomy. The sinus membrane is then perforated when the antrostomy is completed. This implant emplacement, together with adequate implant stability and design, provided enough bone to implant contact (BIC) able to osseointegrate and be responsible for a longterm antrum sealing. Moreover, since alveolar bone buccal to the implant neck is maintained, the risk of late soft tissue complications is minimized. A "tunneltype osteotomy" was also performed in ZAGA type 3 cases where the alveolar bone adopts a triangular, buccally inclined, profile and the maxillary anterior wall is concave. Then, a circular osteotomy of the alveolar bone leaves intact the sinus lining regardless of the maxillary wall curvature. In the case of a tunnel osteotomy preparation, Straumann ZAGA Round zygomatic implant design was chosen to seal the preparation. 

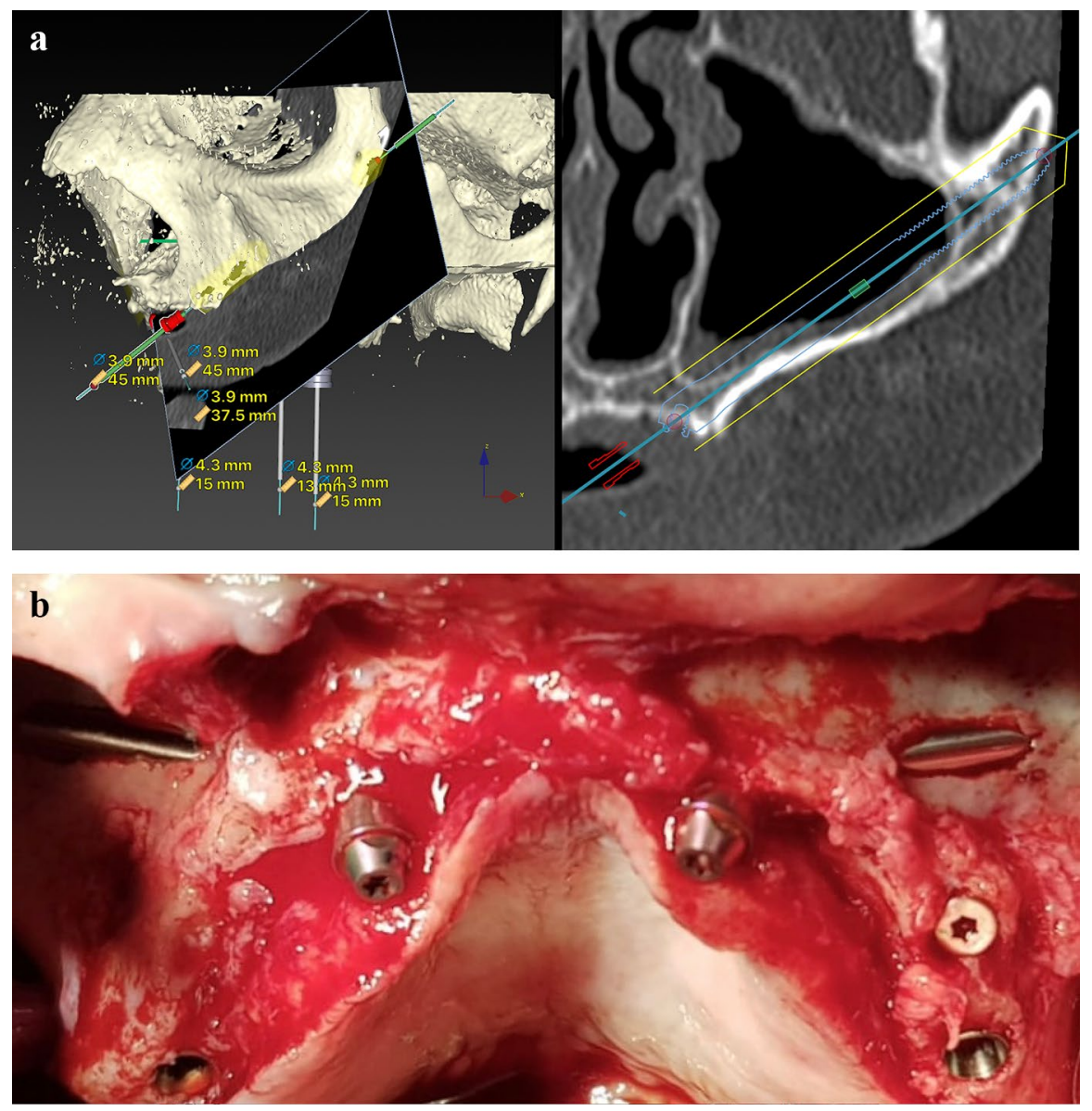

Fig. 6 a Patient CBCT oblique anterior cut showing inadequate bone in the anterior portion preventing placement of regular implants. b The final disposition: regular implants placed in the posterior region and zygomatic implants in the anterior region

In patients with severe resorption and inadequate residual bone architecture at crestal level, as in ZAGA type 4 and some types 2, instead of penetrating the antrum through a thin bone layer, the coronal osteotomy was buccally shifted to prevent future sinus or nasal-oral communication/fistula. The initial preparation is done by placing the ZICZ on the buccal aspect of the residual ridge (Fig. 7). The antrostomy was usually established not at the zygoma itself, but at the zygomatic process of the maxilla, inferior to the zygomaticomaxillary suture, and separated as much as possible from the zygomatic critical zone located were the implant contacts for the first time with the alveolar bone. The final contour of the coronal entrance site is determined by the crestal anatomy and the degree of bone resorption. The ZAGA Concept aims to prevent possible complications. Hence, while performing the first osteotomy close to the crest, the risk of early or late sinus communication and soft 

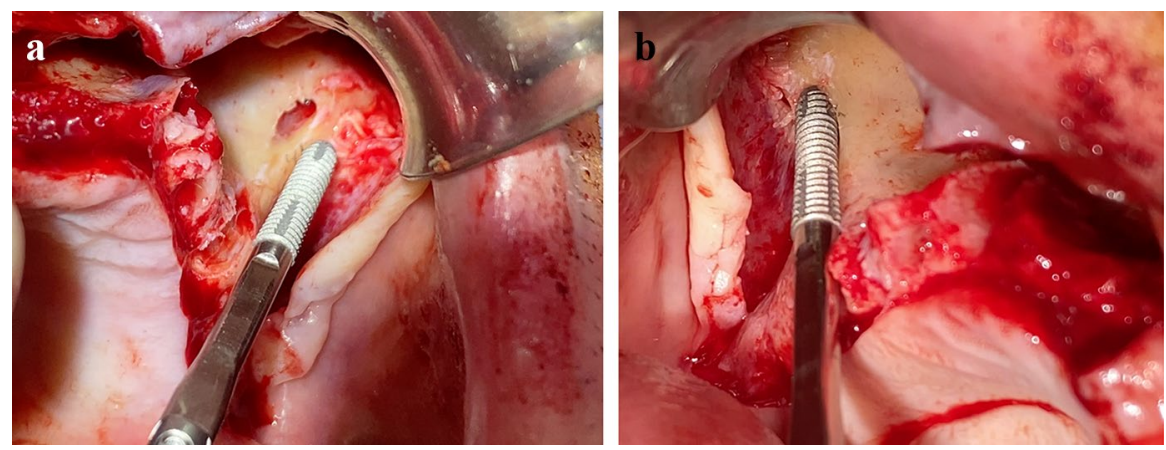

Fig. 7 a Typical entrance in a ZAGA Type IV anatomy from the palatal side of the crest. Implant bed with a 'channel' form osteotomy while maintaining bone thickness and membrane integrity on the facial side of the maxillary wall. b A ZAGA Flat implant on its channel

tissue recession around the implant head or body should be considered. The integrity of the sinus lining at the crest at the ZICZ should be maintained. This type of osteotomy, not capable of providing a complete covering of the implant mid-body and neck, is known by the authors as "channel-type osteotomy". It is a groove made on the coronal alveolar bone, and sometimes also in the lateral maxillary wall and zygomatic buttress. In the case of a channel osteotomy preparation, Straumann ZAGA Flat zygomatic implant design was chosen to avoid soft tissue compression and seal the preparation.

The position, condition, and nature of the initial preparation are strongly associated with both possibilities. An oral-sinus communication can develop over time after a palatal or crestal osteotomy is done through a thin bone of thickness $<2 \mathrm{~mm}$. This lack or late loss of soft tissue or bone sealing around the implant entrance can also be due to:

- a mismatch between the implant diameter and initial osteotomy;

- late infectious peri-implant bone resorption;

- inadequate use of hygiene instruments;

- history and a genetic tendency for periodontitis;

- tobacco consumption;

- physiologic bone atrophy.

The implant should not be inserted too far buccally or too separated from the crestal bone to prevent soft tissue recession due to compression by the implant body. A more buccal position of the implant can cause mucogingival dehiscence. In an extremely atrophic maxilla, where implant sealing is achieved only by soft tissue (at least on its buccal aspect), special attention should be paid to prevent mucogingival dehiscence (Fig. 8). If the above-mentioned complications are unavoidable, prevention of sinus infection should be the operator's highest priority.

In a maxillary atrophy type IV ZAGA case, entry to the sinus is taken as close as possible to the apical entrance point of the zygoma. Additionally, at least 5-10 $\mathrm{mm}$ 
Fig. 8 a Orthoradial cut showing the virtual planning for the anterior implant in an extremely thin wall under the nose at the left lateral/canine position. b Orthoradial cut showing extremely thin wall at the left second premolar first molar position on the same patient. These are the most complex jaws to solve. Special care should be taken to prevent late complications like soft tissue dehiscence or sinus infection. c A ZAGA Round and a ZAGA Flat zygomatic implants were placed in the positions of the left lateral and second premolar, respectively. d The bone volume at the anterior implant neck level is scarce and does not reach the ideal length to appropriately sink the implant head. Placing the implant deeper will increase the risk of nose penetration or late sinus infection. In such cases, soft tissue dehiscence should be predicted and prevented. Additional use of the ZAGA Scarf Graft is advised. e Orthoradial cut showing the final position of the anterior implant at the 1-year follow-up. Note the total transparency of the maxillary sinus. f Orthoradial cut showing the final position of the posterior implant. Note the total transparency of the maxillary sinus at the 1-year follow-up
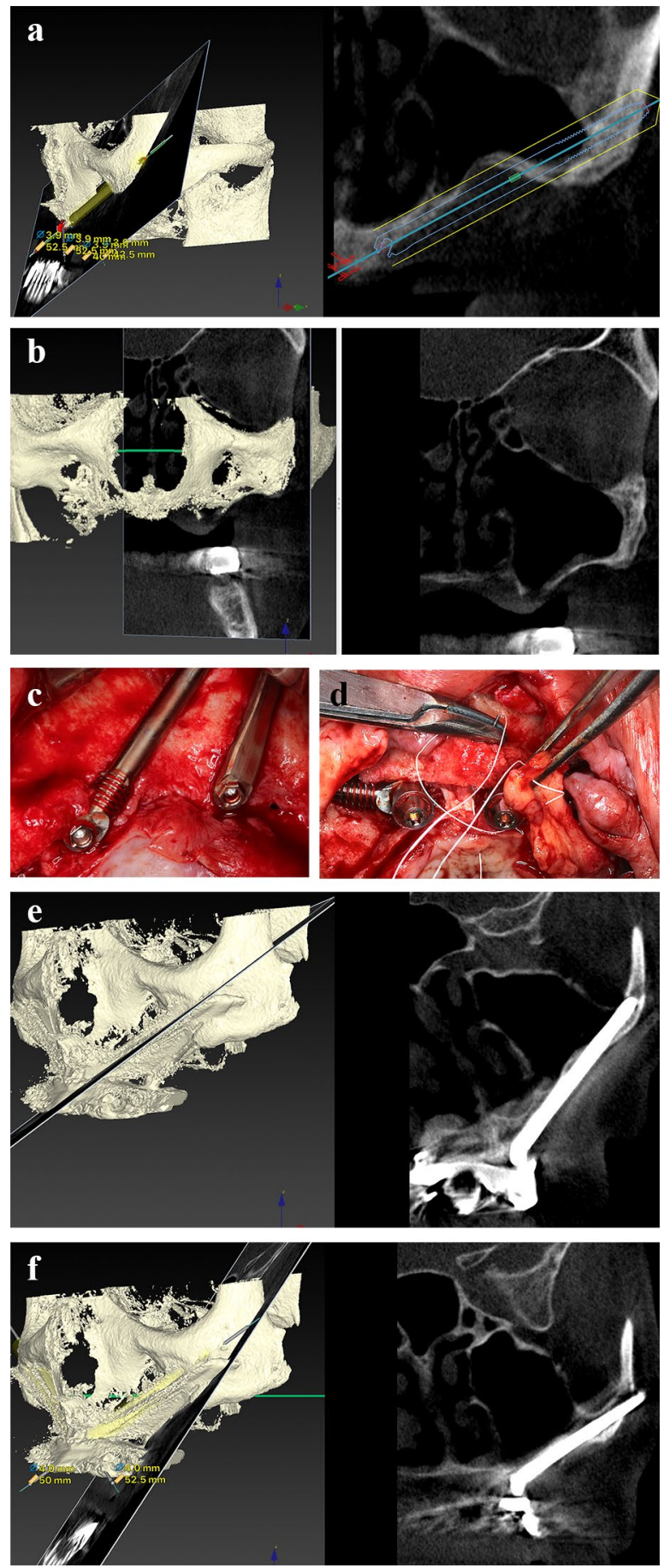


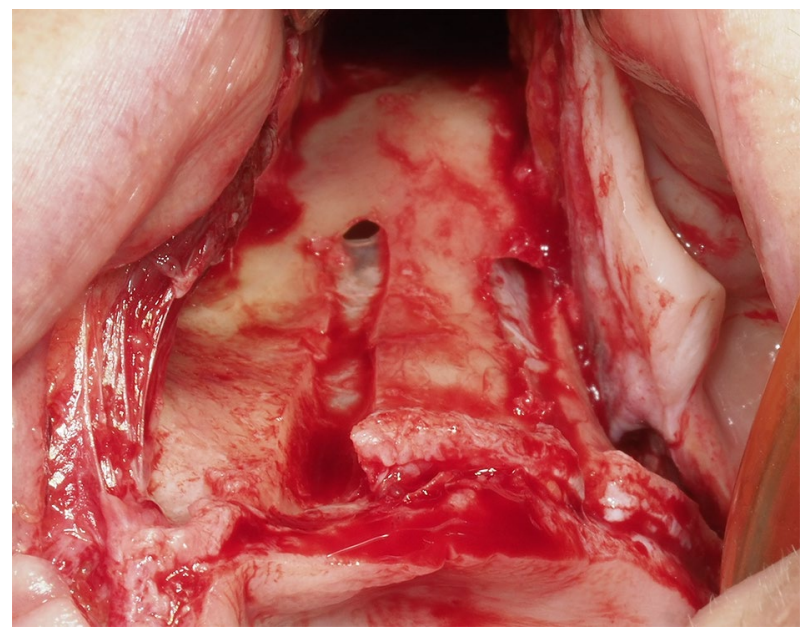

Fig. 9 View of two beds of 'ZAGA-Channel' zygomatic osteotomy, customized in the most conservative way, maintaining more than $10 \mathrm{~mm}$ (as much as possible) of bone in the maxillary wall. Note the apical zygomatic entrance does not respect membrane integrity

of residual bone and intact membrane at the crest level of the maxillary anterior wall should be maintained lateral to the implant neck (Fig. 9).

The ZAGA 0 to IV classification describes the association of the zygomatic buttress-alveolar crest regions including alternatives for the various ZAGA implant paths. It aids the dentist in understanding inter-individual and intra-individual anatomic variations. When the residual bone at the sinus floor level has adequate thickness and width (minimum: $4 \mathrm{~mm}$ height, $6 \mathrm{~mm}$ width) in a patient without a history of periodontitis, the position of the entry point should be close to the middle portion of the crest with an intra-sinus starting path of the implant if the maxillary wall is flat or convex. When the crestal bone height or thickness is inadequate, the alveolar entrance point should be shifted buccally, regardless of the maxillary wall curvature. Based on the maxillary wall concavity and the height of pristine bone, the osteotomy is shaped like a tunnel or canal (Figs. 5 and 10).

In cases where the alveolar architecture is insufficient, the main objective is to establish an implant trajectory that:

- shifts the antrostomy at least $15 \mathrm{~mm}$ away (as far as possible) from the coronal region of the implant (Figs. 3, 7, 9, 10);

- keeps a minimum of $5 \mathrm{~mm}$ (as much as possible) of alveolar bone in contact with the implant neck (Figs. 3, 7, 9, 10);

- houses the implant body submerged as much as possible into the alveolar bone for avoiding soft tissue compression while also maintaining sinus membrane integrity (Figs. 3, 7, 9, 10). 

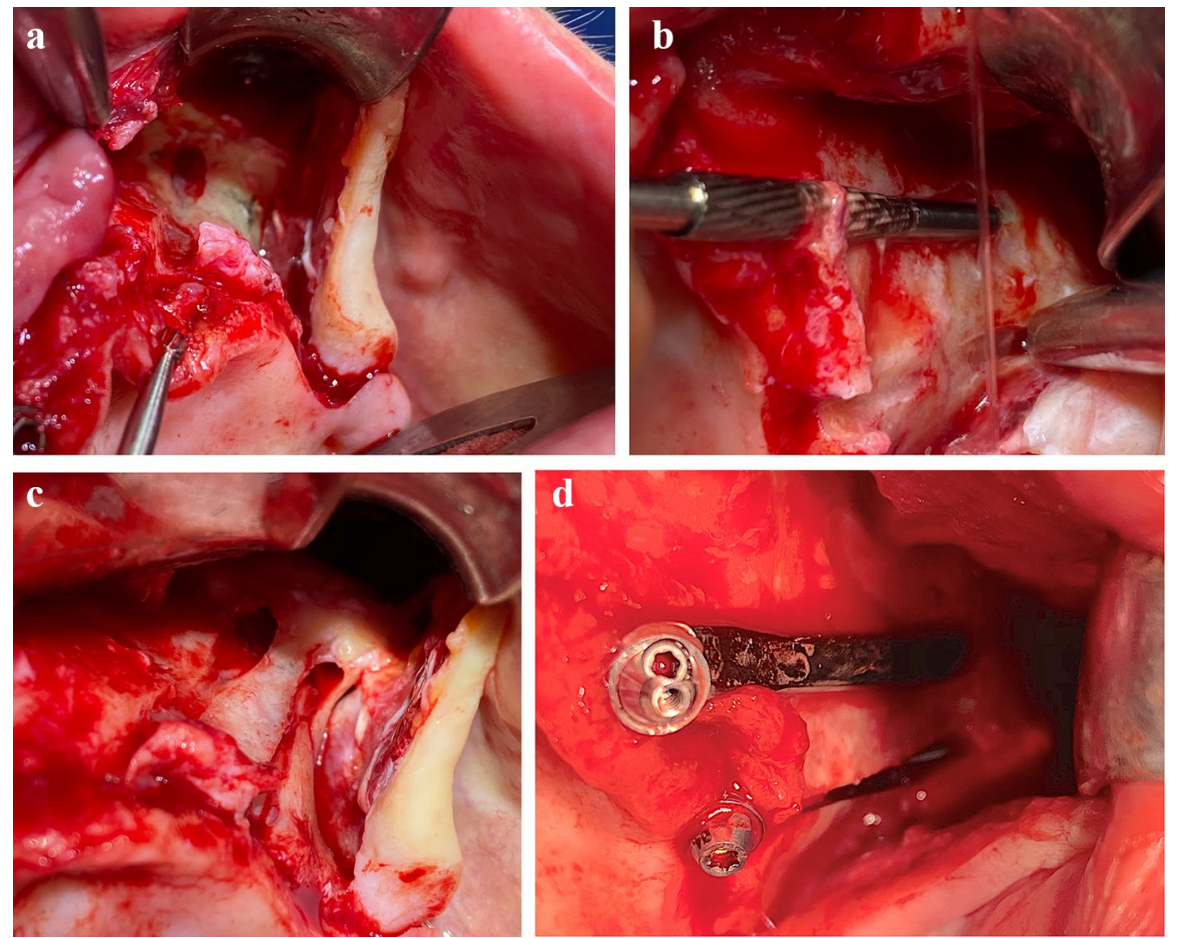

Fig. 10 a Entrance on the palatal side to create an implant bed to bury the implant to the maximum depth by preventing soft tissue dehiscence and while maintaining maximum possible bone thickness at the crestal and buccal side of the maxillary wall. b Enlarging the anterior maxilla to accommodate the implant neck. c. The two ZAGA minimally invasive osteotomies were performed to accommodate the implant profile. Prevention of late sinus complications is crucial. d Anterior and posterior Straumann ZAGA Flat implant placement from the palatal side through a 'ZAGA Channel' osteotomies. Profile view. Both osteotomies accommodate the implant head thickness

\section{B. Determination of the apical zygomatic entrance point}

The zygomatic implant gains its main anchorage when the zygomatic bone is perforated twice on its facial cortical side. An oblique lateralized path of the implant should be aimed for whenever possible. As per the ZAGA protocol, the key factors while selecting the anterior maxillary or zygomatic cortical entry point of the implant are:

- number of implants to be inserted;

- amount of zygomatic bone left above the apical threads of the implant (Fig. 11);

- the curvature of the zygomatic process.

At this surgical stage, iatrogenic zygomatic bone fracture and interference with structures of the orbital fossa should be avoided (Fig. 11). 


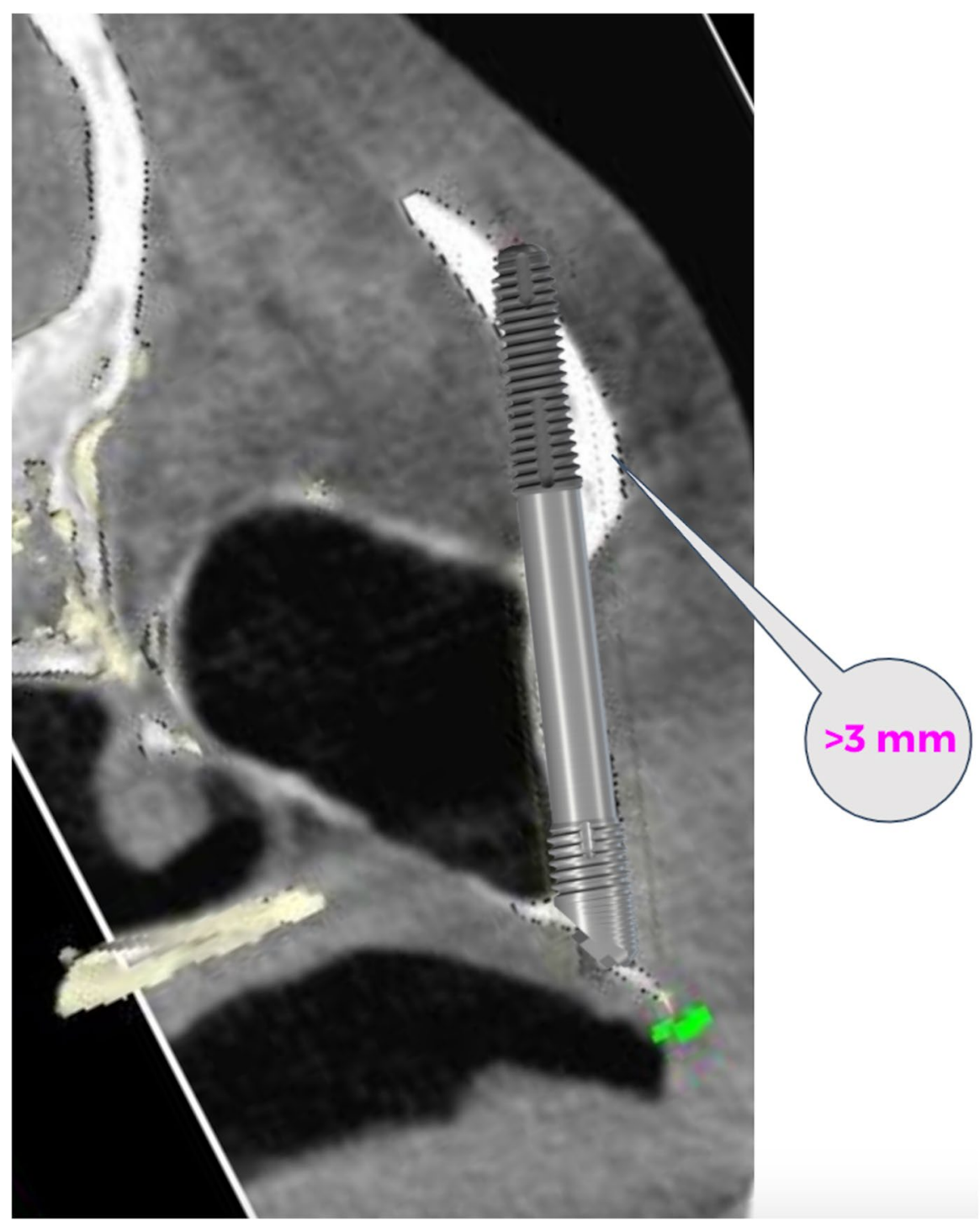

Fig. $11 \mathrm{CT}$ scan and virtual simulation showing the minimum amount of bone $(3 \mathrm{~mm})$ that should be left over the implant to prevent zygomatic bone fracture during drilling

Two prevalent situations associated with the number of zygomatic implants to be inserted are:

- In the case of posterior atrophy with adequate bone in the inter-canine region, insertion of a single zygomatic implant on each side is required. A more upright implant position that places the implant head as distally as possible is desired to reduce the posterior cantilever. However, when the first molar site is used rather 
than the second premolar site, the final drill exit can be too close to the orbit. Special attention should be paid to prevent orbital perforation and damage to the orbital contents.

- In the case of anterior and posterior atrophy, a Quad approach that includes the placement of two implants in each zygomatic bone is commonly performed. Utmost care should be taken as one of the implants needs to be inserted close to the orbit. Exploration of the orbital fossa and infra-orbital arc is essential before the drilling procedure.

Following the ZAGA Concept of prevention of complications, the authors prefer contemporary narrow implant designs with an apical diameter of 3,4 mm. These designs need a smaller initial osteotomy and preserve more bone volume between implants, which in turn decreases the risk of zygoma bone fractures. Two main aspects to be taken into consideration while achieving zygomatic implant stability are:

- At the coronal level: improved stability can be achieved by splinting all inserted implants with a rigid prosthetic framework.

- At implant apex: zygomatic implant stability depends on the volume of bone-toimplant contact obtained during implant placement at the zygomatic level, which in turn depends on the path from the maxillary or zygomatic inferior entry point up to the upper zygomatic cortical exit point.

As per the ZAGA protocol, the osteotomy at the zygomatic level should have two features:

- Perforate the facial side of the zygomatic bone twice (inferior and superior aspect).

- Leave a minimum of $3 \mathrm{~mm}$ of bone above the threaded apical portion of the implant (Fig. 11).

The zygomatic path of the osteotomy requires maximum implant stabilization. This is achieved by perforating the facial aspect of the zygoma twice, once at the inferior entry point and then at the superior exit point. After deciding the alveolar implant position, the zygomatic entrance is decided according to the zygoma process curvature. The more curved the zygomatic process, the further from the alveolar bone will be the antrostomy position, whereas the less pronounced the facial zygomatic curvature, the closer to the alveolar process will be the antrostomy. The zygomatic osteotomy can include posterior perforation of the zygomatic bone into the temporal fossae, partial implant path on the posterior temporal fossae, zygomatic upper posterior entrance from the sinus cavity, or temporal fossae, and final zygomatic upper anterior cortical exit.

For prevention of a drilling-related zygoma fracture, it is essential to strategically position the zygomatic entry point to maintain at least $3 \mathrm{~mm}$ of residual bone above the apical end of the zygomatic implant. The narrower the implant diameter, the more conservative and less invasive will be the necessary osteotomy (Fig. 11). 


\section{Determination of the implant path}

After defining the coronal entry point on the crestal bone, the bur points toward the zygomatic bone. The ZAGA technique does not require an antrostomy before the zygomatic osteotomy in most cases. The aim of placing zygomatic implants is to establish an implant bed that complements the implant body. Matching the osteotomy to the implant configuration eliminates the requirement of a lateral window and preserves the maximum amount of bone at the crest and the maxillary lateral wall level (Fig. 10). During the surgery, no slot or window is created. Alternatively, a direct apical maxillary or zygomatic osteotomy is done that complements the implant path. The factors dictating the apical osteotomy site and the implant direction are:

- The benefit of a mucoperiosteal flap allows surgical control of the anatomic limits and structures, including the superior zygomatic rim and the posterior wall of the maxillary sinus facing the temporal fossae.

- Use of a retractor positioned at the superior zygomatic rim to aid the surgeon in:

- enlarging the field of vision;

- guiding implant direction deviations;

- protecting soft tissue injury from the drill during final perforation of the upper facial zygomatic cortical bone.

When the coronal and apical points are connected by inserting an implant, the implant body complements and seals the osteotomy eliminating the requirement of bone grafts.

We are conscious that the proposal of not making a maxillary window prior to the osteotomy preparation can create the sensation of working blind. However, this is not the case for different reasons. Total control of the surgical field boundaries is obtained provided an adequate flat is raised (Fig. 2a, b). Then, a modified retractor instrument will be located on the angle formed by the temporal and frontal apophasis of the zygoma. Indeed, adequate flap extension and retractor position can satisfactorily expose the limits of the intervention. Precise knowledge of the specific zygomatic, maxillary and crestal anatomy is achieved thanks to the pre-surgical CBCT. Indeed, the entrance and emerging alveolar and zygomatic points are virtually planned to follow the ZAGA protocol before the surgery. Before the drilling procedure, the intended ZICZ and the antrostomy points are transferred to the surgical field using a marker. In the case of a ZAGA type 0 maxilla showing an adequate amount of remaining sinus floor (i.e., $>4 \mathrm{~mm}$ height), the zygomatic entrance cannot be directly seen since an intra-sinus path is planned. Then, a guiding line, externally joining the alveolar entrance with the planned zygomatic entrance, is drawn to provide drilling orientation to the surgeon. Ideally, a rehearsal surgery is performed in a 3-D model (3-D models can be printed by sending the DICOM images to ct@ zagacenters.com and completing a form at www.zagacenters.com) (Fig. 12).

The implant osteotomy is achieved by joining the ZICZ and the AZ sites once they have been identified. Implant path is obtained by preparing a particular 


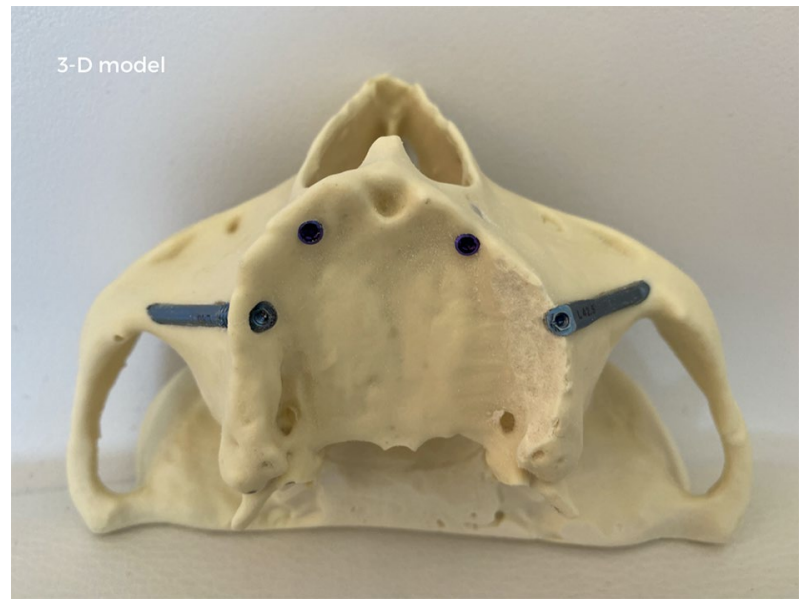

Fig. 12 The 3-D model from a real patient shows a rehearsal surgery. Two Straumann BLX implants were installed on the anterior zone. A Straumann ZAGA Round zygomatic implant is located on the ZAGA 3 crest of the right side. A Straumann ZAGA Flat zygomatic implant is found on the left arch. The 3-D model is obtained by sending DICOM images to ct@zagacenters.com and filling an order form at www.zagacenters.com

osteotomy in as less traumatic as possible manner with no necessity for a previous "window" osteotomy. As a result, maximal BIC is obtained along the residual alveolar crest, the maxillary wall, and the zygomatic bone. Moreover, the implant profile will totally seal the minimally invasive osteotomy.

The ZICZ is visually determined using the aforementioned parameters. The zygomatic entrance point can be directly seen in almost every case (unless we use an intra-sinus path). No matter how large the "window" may be, usually, through a maxillary "window osteotomy" it is not possible to see the outer side upper emergence point of the drill. The hypothetical direct visualization of the upper inner perforation of the drill has been claimed to be possible by placing a rectangular "window" at the level of the zygomatic process of the maxilla. However, what we "see" is the drill traversing the window but not the final upper spot to be perforated. Moreover, by using a rectangular upper window, we may lose some mechanical properties of the implant (i.e., exposed apical threads diminishing the stabilization power of the implant).

\section{ZAGA implant trajectories}

Owing to anatomical variations, a variety of positions of zygomatic implants can be seen in relation to the maxillary wall from an intra-sinus path to a completely extrasinus path. As per the ZAGA protocol, implant insertion can be classified into five types based on the implant path:

ZAGA type 0: is characterized as:

- Having a very flat anterior maxillary wall. 

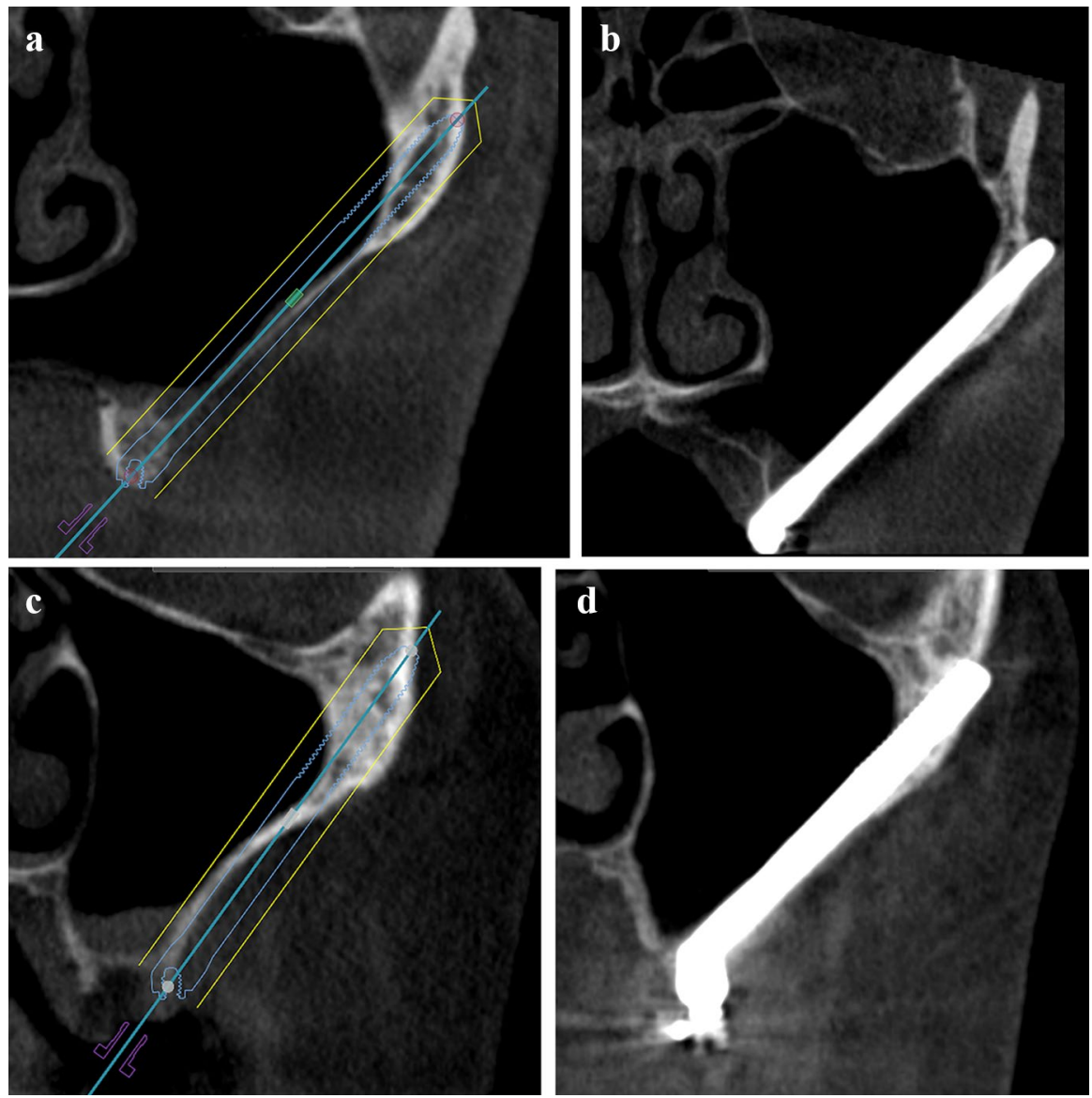

Fig. 13 a Tomographic section showing a straight maxillary ZAGA Type 0 wall. Bone height under the sinus is about $4 \mathrm{~mm}$ thick. Virtual placement of implant through a sinus floor as per the ZAGA Type 0 protocol. b Tomographic section showing the post-operative implant status planned in (a) at the checkup. c Computed tomography shows a straight maxillary wall. Bone height under the sinus is about $1-2 \mathrm{~mm}$ thick. Preservation of bone crest integrity is mandatory to prevent later oro-antral communication. Accordingly, the implant was placed extra-sinus, buccal to the crest, converting the case into a ZAGA Type IV protocol. d Tomographic section showing the post-operative implant status as planned in (c) at the checkup

- Implant head is positioned in the pristine bone if the available bone is at least $4 \mathrm{~mm}$ high and $6 \mathrm{~mm}$ wide (Fig. 13a,b).

- A meticulously underprepared tunnel-type osteotomy through $4 \mathrm{~mm}$ of crestal bone height and the sinus floor lining facilitates implant stabilization, periimplant bone sealing, and repair of sinus membrane within a duration of 2-3 weeks.

- In a patient with inadequate bone or a history of smoking or periodontitis, the procedure should be changed to an exteriorized approach to position the antrostomy site away from the bone crest (Figs. 13 and 14). 

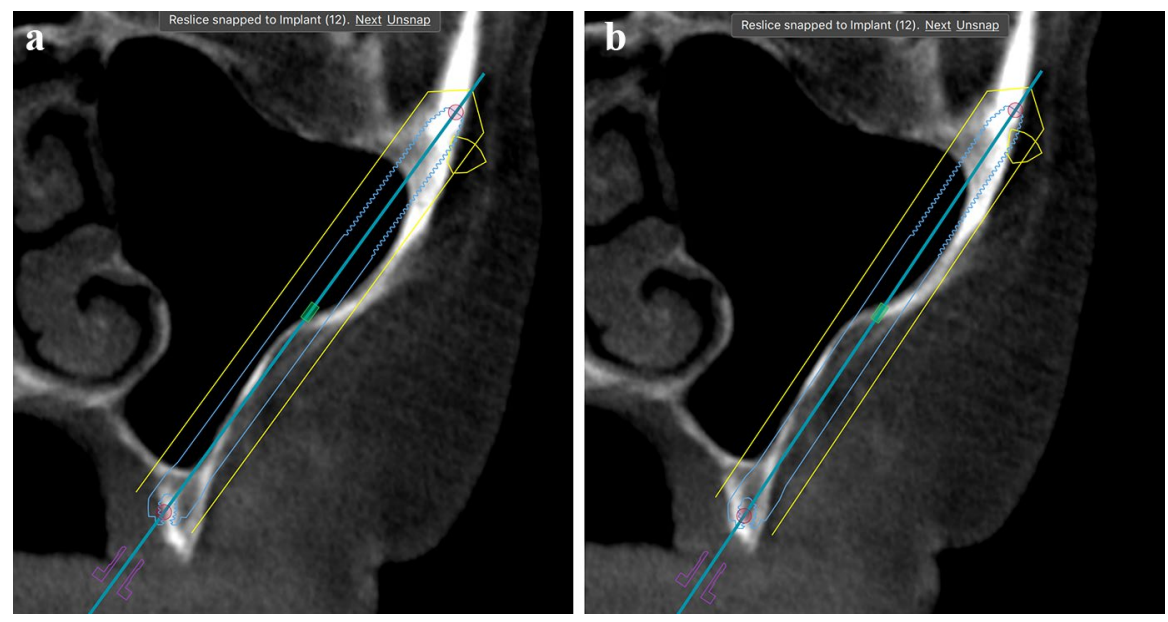

Fig. 14 a Computed tomography showing a slightly curved maxillary wall. Bone height under the sinus cannot properly house the zygomatic implant neck. Virtual placement of implant through the sinus floor and crest. Late sinus-related complications should be expected due to the poor thickness of the sinus floor on its palatal side. b Computed tomography showing the same maxillary wall as (a). Since bone height under the sinus is reduced and with the goal of long-term preservation of bone crest integrity, the implant was placed extra-sinusal, buccal to the crest, converting the case into a ZAGA Type IV protocol

- The implant body has an intra-sinus path.

- Implant to bone contact is seen at the alveolar crest and zygomatic bone and, rarely, at the lateral sinus wall.

- A Straumann ZAGA Round zygomatic implant section is used to close the osteotomy and to maintain bone at the sinus floor.

ZAGA type I: has a combined intra- and extra-sinus path with most of the implant body located intra-sinusally:

- Has a slightly concave anterior maxillary wall.

- The implant head is positioned on the alveolar crest if the available bone around the implant can be maintained at a height of at least $4 \mathrm{~mm}$. The intrasinus path technique along with a window antrostomy and subsequent grafting is deemed more invasive and riskier.

- The tunnel-type osteotomy is performed by drilling slightly through the wall.

- Most of the implant body has an intra-sinus path, but can still be seen through the wall.

- A prior window osteotomy is not commonly needed as the residual alveolar crest is slightly, buccally tilted facilitating an exteriorized approach.

- Implant contacts bone at the alveolar crest, lateral sinus wall, and zygomatic bone (Fig. 6b).

- A Straumann ZAGA Round zygomatic implant section is used to close the osteotomy and to maintain bone at the sinus floor. 
ZAGA type II: has a combined extra- and intra-sinus path with most of the implant body being located extra-sinusally:

- Has a concave anterior maxillary wall.

- The implant head is positioned on the alveolar crest.

- Osteotomy should be made by drilling through the wall complementing the implant shape. Usually, a channel-type osteotomy will be obtained

- There is no prior sinus window or slot.

- Most of the implant body has an extra-sinus path with the implant being seen through the wall. In a patient with a history of smoking or periodontitis or with inadequate palatal bone height around the implant (Fig. 14a), the implant path should be placed with an exteriorized approach to position the antrostomy away from the entry point (Fig. 14b).

- Implant-bone contact can be seen at the alveolar crest, zygomatic bone, and lateral sinus wall. (Fig. 4 anterior implant).

- A Straumann ZAGA Flat zygomatic implant section is used to close the osteotomy and to maintain bone at the sinus floor.

ZAGA type III has an in (alveolar)-out (extra-sinus)-in (zygomatic) path with other characteristics:

- Having a very concave anterior maxillary wall.

- Implant head is located on the alveolar crest.

- The tunnel-type osteotomy is performed using a drill by following a trajectory from the palatal to the upper buccal alveolar bone. Thereafter, the implant body leaves the concave part of the anterior sinus wall and penetrates the zygoma.

- There is no prior sinus window or slot.

- Most of the implant body has an anterior extra-sinus path.

- The middle portion of the implant body does not contact the most concave section of the wall.

- Implant-bone contact is seen at the alveolar crest and the zygomatic bone. (Fig. 3c anterior implant).

- A Straumann ZAGA Round zygomatic implant section is used to close the osteotomy and to maintain bone at the sinus floor.

ZAGA type IV has an extra-maxillary path with the following features:

- There is severe vertical and horizontal atrophy in the maxillary and alveolar bones.

- The location of the implant head is buccal to the alveolar crest. A channeltype osteotomy at this level was made to preserve maximum bone volume at the crestal and coronal maxillary wall level.

- The drill exits at the apical zygomatic entrance point by following an extrasinus path. 
- There is no prior slot or window. Most of the implant body has an extra-sinus or extra-maxillary path. Only the apical portion of the implant is surrounded by bone.

- The implant-bone contacts are seen at the zygoma and lateral sinus wall (Fig. 3c posterior implant).

- A Straumann ZAGA Flat zygomatic implant section is used to close the osteotomy and to maintain the bone at the sinus floor.

The ZAGA protocol's main objective is to establish optimal soft tissue-implant sealing. Hence, immediate placement of final abutments is advised. Additionally, it is also recommended to place and equilibrate an immediate provisional prosthesis within the initial $24 \mathrm{~h}$. The provisional prosthesis should not have distal cantilevers to minimize the applied load. For bone-implant healing, a soft diet is recommended for a period of about 3 months.

\section{Pitfalls \& complications}

- The potential complications of performing the first osteotomy close to the crest are risks of early or late sinus communication and soft tissue recession around the implant head or body.

- A more buccal implant position can cause mucogingival dehiscence.

Open Access This article is licensed under a Creative Commons Attribution 4.0 International License, which permits use, sharing, adaptation, distribution and reproduction in any medium or format, as long as you give appropriate credit to the original author(s) and the source, provide a link to the Creative Commons licence, and indicate if changes were made. The images or other third party material in this article are included in the article's Creative Commons licence, unless indicated otherwise in a credit line to the material. If material is not included in the article's Creative Commons licence and your intended use is not permitted by statutory regulation or exceeds the permitted use, you will need to obtain permission directly from the copyright holder. To view a copy of this licence, visit http://creativecommons.org/licen ses/by/4.0/.

\section{Further Reading}

1. J. Chow (ed) (2020) The zygoma anatomy-guided approach: ZAGA-A patient-specific therapy concept for the rehabilitation of the atrophic maxilla. In: Zygomatic implants. Springer. https://doi.org/ 10.1007/978-3-030-29264-5_5

2. Chow J, Wat P, Hui E, Lee P, Li W (2010) A new method to eliminate the risk of maxillary sinusitis with zygoma implants. Int J Oral Maxillofac Implants 25:1233-1240

3. Davó R, Felice P, Pistilli R, Barausse C, Marti-Pages C, Ferrer-Fuertes A et al (2018) Immediately loaded zygomatic implants vs conventional dental implants in augmented atrophic maxillae: 1-year post-loading results from a multicentre randomised controlled trial. Eur J Oral Implantol 11:145-161 
4. Felice P, Barausse C, Davó R, Pistilli R, Marti-Pages C, Ferrer-Fuertes A et al (2020) Immediately loaded zygomatic implants versus conventional dental implants in augmented atrophic maxillae: three-year post-loading results from a multicentre randomised controlled trial. Clin Trials Dentistry 02:5-25

5. Brånemark P-I, Gröndahl K, Ohrnell L-O, Nilsson P, Petruson B, Svensson B et al (2004) Zygoma fixture in the management of advanced atrophy of the maxilla: technique and long-term results. Scand J Plast Reconstr Surg Hand Surg 38:70-85

6. Jensen OT, Adams M, Cottam JR, Ringeman J (2013) Occult peri-implant oroantral fistulae: posterior maxillary peri-implantitis/sinusitis of zygomatic or dental implant origin. Treatment and prevention with bone morphogenetic protein-2/absorbable collagen sponge sinus grafting. Int J Oral Maxillofac Implants 28:e512-520

7. Aparicio C, López-Piriz R, Albrektsson T (2020) ORIS criteria of success for the zygoma-related rehabilitation: the (revisited) zygoma success code. Int J Oral Maxillofac Implants 35:366-378

8. Aparicio C, Manresa C, Francisco K, Aparicio A, Nunes J, Claros P et al (2014) Zygomatic implants placed using the zygomatic anatomy-guided approach versus the classical technique: a proposed system to report rhinosinusitis diagnosis. Clin Implant Dent Relat Res 16:627-642

9. Aparicio C, Manresa C, Francisco K, Claros P, Alández J, González-Martín O et al (2000) Zygomatic implants: indications, techniques and outcomes, and the zygomatic success code. Periodontol 2014(66):41-58

10. Aparicio C (2011) A proposed classification for zygomatic implant patient based on the zygoma anatomy guided approach (ZAGA): a cross-sectional survey. Eur J Oral Implantol 4:269-275

11. Aparicio C (ed) (2012) Zygomatic Implants: the Anatomy-guided Approach, 1st edn. Quintessence Pub Co

12. Nkenke E, Hahn M, Lell M, Wiltfang J, Schultze-Mosgau S, Stech B et al (2003) Anatomic site evaluation of the zygomatic bone for dental implant placement. Clin Oral Implants Res 14:72-79

13. Aparicio C, Ouazzani W, Aparicio A, Fortes V, Muela R, Pascual A et al (2010) Immediate/Early loading of zygomatic implants: clinical experiences after 2 to 5 years of follow-up. Clin Implant Dent Relat Res 12(Suppl 1):e77-82

14. Jensen OT (2014) Complete arch site classification for all-on-4 immediate function. J Prosthet Dent 112:741-51.e2

15. Duarte LR, Filho HN, Francischone CE, Peredo LG, Brånemark P-I (2007) The establishment of a protocol for the total rehabilitation of atrophic maxillae employing four zygomatic fixtures in an immediate loading system-a 30-month clinical and radiographic follow-up. Clin Implant Dent Relat Res 9:186-196

16. Stiévenart M, Malevez C (2010) Rehabilitation of totally atrophied maxilla by means of four zygomatic implants and fixed prosthesis: a 6-40-month follow-up. Int J Oral Maxillofac Surg 39:358-363

17. Davó R, Pons O (2015) 5-year outcome of cross-arch prostheses supported by four immediately loaded zygomatic implants: a prospective case series. Eur J Oral Implantol 8:169-174

18. Freedman M, Ring M, Stassen LFA (2015) Effect of alveolar bone support on zygomatic implants in an extra-sinus position-a finite element analysis study. Int J Oral Maxillofac Surg 44:785-790

19. Davo R, Malevez C, Rojas J (2007) Immediate function in the atrophic maxilla using zygoma implants: a preliminary study. J Prosthet Dent 97:S44-51

20. Aparicio C, Antonio S (2020) Zygoma anatomy-guided approach "scarf graft" for prevention of soft tissue dehiscence around zygomatic implants: technical note. Int J Oral Maxillofac Implants 35:e21-e26

Publisher's Note Springer Nature remains neutral with regard to jurisdictional claims in published maps and institutional affiliations. 


\section{Authors and Affiliations}

Carlos Aparicio ${ }^{1}$ (1) Antonio Olivo ${ }^{2} \cdot$ Victor de Paz $^{3} \cdot$ Daniel Kraus $^{4}$. Manuel Martin Luque ${ }^{5} \cdot$ Eduardo Crooke $^{6} \cdot$ Peter Simon $^{7} \cdot$ Madalina Simon $^{7}$. Jose Ferreira ${ }^{8} \cdot$ Andre Sakima Serrano $^{9} \cdot$ Jan Peter $~ \mathrm{Ig}^{9} \cdot$ Arturo Bilbao $^{10}$. Alberto Fernandez ${ }^{11}$. Pedro Guitián ${ }^{12}$. Jay Neugarten ${ }^{13}$

Antonio Olivo

a.olivo@medicalcenterpadova.it

Victor de Paz

vdepaz@gmail.com

Daniel Kraus

dkraus@dr-kraus.com

Manuel Martin Luque

manuel.martin@clinicasioc.com

Eduardo Crooke

duitocrooke@gmail.com

Peter Simon

dres.simon@me.com

Madalina Simon

dres.simon@me.com

Jose Ferreira

jose.j.r.ferreira.299@gmail.com

Andre Sakima Serrano

andresakima@gmail.com

Jan Peter Ilg

jpilg@uol.com.br

Arturo Bilbao

arturobilbao@secom.org

Alberto Fernandez

direccion@clinicafernandez.es

Pedro Guitián

pedro@clinicaguitian.com

Jay Neugarten

drNeugarten@nycoms.com

1 ZAGA Center Barcelona, Director Zygomatic Unit at Hepler Bone Clinic, Roman Macaya 22-24, 08022 Barcelona, Spain

2 ZAGA Center Triveneto - Medical Center Padova, Padua, Italy

3 ZAGA Center Madrid - Instituto Maxilofacial IMA, Barcelona, Spain

4 ZAGA Center Frankfurt Mainz - Dr. Kraus Zahnärzte \& Implantatklinik, Mainz, Germany

5 ZAGA Center Canary Islands - IOC Clínica Dental, Vecindario, Spain 
6 ZAGA Center Costa del Sol - Clínica Dental Crooke \& Laguna, Marbella, Spain

7 ZAGA Center Stuttgart - Zahnarztpraxis Dres. Simon, Stuttgart, Germany

8 ZAGA Center Braga - DIMD- Diagnóstico Integrado em Medicina Dentária, Braga, Portugal

9 ZAGA Center São Paulo - CA.SA Odontologia, São Paulo, Brazil

10 ZAGA Center A Coruña y Lugo - AB Cirugía Oral y Maxilofacial, A Coruña, Spain

11 ZAGA Center Ibiza - Clínica Fernández, Ibiza, Spain

12 ZAGA Center Pontevedra \& Orense - Clínica Guitián, Pontevedra, Spain

13 ZAGA Center New York - The New York Center for Orthognathic and Maxillofacial Surgery, New York, USA 